\title{
Impact of cholesterol metabolism and the LXR $\alpha$-SREBP-1c pathway on nonalcoholic fatty liver disease
}

\author{
MAKOTO NAKAMUTA $^{1,2}$, TATSUYA FUJINO ${ }^{2}$, RYOKO YADA ${ }^{2}$, MASAYOSHI YADA ${ }^{3}$, \\ KENICHIRO YASUTAKE ${ }^{2}$, TSUYOSHI YOSHIMOTO $^{1}$, NAOHIKO HARADA $^{1}$, NOBITO HIGUCHI $^{3}$, \\ MASAKI KATO $^{3}$, MOTOYUKI KOHJIMA ${ }^{3}$, AKINOBU TAKETOMI ${ }^{4}$, YOSHIHIKO MAEHARA $^{4}$, \\ MANABU NAKASHIMA ${ }^{5}$, KAZUHIRO KOTOH ${ }^{3}$ and MUNECHIKA ENJOJI ${ }^{5}$
}

\begin{abstract}
${ }^{1}$ Department of Gastroenterology, ${ }^{2}$ Clinical Research Center, Kyushu Medical Center, National Hospital Organization; Departments of ${ }^{3}$ Medicine and Bioregulatory Science, ${ }^{4}$ Surgery and Science, Graduate School of Medical Sciences, Kyushu University; ${ }^{5}$ Department of Clinical Pharmacology, Faculty of Pharmaceutical Sciences, Fukuoka University, Fukuoka, Japan
\end{abstract}

Received December 1, 2008; Accepted January 19, 2009

DOI: 10.3892/ijmm_00000170

\begin{abstract}
We previously studied fatty acid metabolism in the liver of nonalcoholic fatty liver disease (NAFLD) and reported the activation of the LXR $\alpha$-SREBP-1c pathway in hepatocytes. $\mathrm{LXR} \alpha$ regulates cholesterol metabolism as well as fatty acid metabolism, and its agonistic ligands are oxysterols. Moreover, there is some evidence that excess cholesterol intake is involved in the onset of NAFLD. Therefore, in this study, we examined the expression of cholesterol metabolismassociated genes in the NAFLD liver by real-time PCR. Expression of $\mathrm{LXR} \alpha$ and ACAT1 was up-regulated in NAFLD and this was more noticeable in non-obese rather than in obese patients. Although the expression of the LDL receptor, which acts on cholesterol uptake, and of SREBP-2, a positive key regulator of cholesterol, was suppressed, the
\end{abstract}

Correspondence to: Dr Munechika Enjoji, Department of Clinical Pharmacology, Faculty of Pharmaceutical Sciences, Fukuoka University, 8-19-1 Nanakuma, Jonan-ku, Fukuoka 8140180, Japan

E-mail: enjoji@adm.fukuoka-u.ac.jp

Abbreviations: ABC, ATP binding cassette; ACAT, acyl-CoA: cholesterol acyltransferase; AMPK, AMP-activated protein kinase; ER, endoplasmic reticulum; FDPS, farnesyl-diphosphate synthase; HMGR, HMG-CoA reductase; HMGS, HMG-CoA synthase; Insigs, insulin-induced genes; IRS-1, insulin receptor substrate-1; LDLR, LDL receptor; LXR $\alpha$, liver X receptor $\alpha$; MTP, microsomal triglyceride transfer protein; NAFLD, nonalcoholic fatty liver disease; NASH, nonalcoholic steatohepatitis; NPC1L1, NiemannPick C1 like 1; PPAR $\alpha$, peroxisome proliferator activated receptor $\alpha$; SREBP, sterol regulatory element-binding protein; Scap, SREBPcleavage activating protein; SS, squalene synthase

Key words: nonalcoholic fatty liver disease, cholesterol, liver $\mathrm{X}$ receptor $\alpha$, fatty acid expression of enzymes that promote cholesterol synthesis was uniformly increased in NAFLD. Gene expression of apoB100 and microsomal triglyceride transfer protein, which are associated with VLDL secretion, and ABCG5, which is involved in cholesterol excretion, was significantly elevated in NAFLD. Because cholesterol accumulates in hepatocytes in NAFLD liver, cholesterol uptake and synthesis should be physiologically down-regulated. However, cholesterol synthesis was activated in NAFLD liver, meaning that cholesterol metabolism is dysregulated in NAFLD. Overproduction of cholesterol may lead to an increased level of oxysterols, activation of LXR $\alpha$ and SREBP-1c, and enhanced fatty acid synthesis.

\section{Introduction}

Nonalcoholic fatty liver disease (NAFLD) is one of the most frequent causes of liver dysfunction, and its incidence is markedly increasing. More than $10 \%$ of patients with NAFLD develop nonalcoholic steatohepatitis (NASH), characterized by inflammatory cell-infiltration and ballooning of hepatocytes in the liver, which eventually causes liver cirrhosis and hepatocellular carcinoma (1). However, the mechanisms involved in the pathogenesis of NAFLD in humans have not been thoroughly investigated. To control the initiation and progression of NAFLD, it is necessary to understand the precise mechanism of regulation of lipogenesis in the human liver; therefore, fatty acid metabolism in hepatocytes is of considerable interest.

We comprehensively investigated the hepatic expression pattern of fatty acid metabolism-associated genes in patients with NAFLD (2-6). We found that despite increased uptake of free fatty acids and intracellular accumulation of fatty acids and triglycerides, the expression of sterol regulatory elementbinding protein-1c (SREBP-1c), a key positive regulator of fatty acid synthesis, was still up-regulated, and the expression of AMP-activated protein kinase (AMPK), a negative regulator of fatty acid synthesis, was down-regulated. This led to the activation of fatty acid oxidation, reactive oxygen species production, and synthesis of phospholipids and 
triglycerides. This expression pattern of SREBP-1c and AMPK in NAFLD liver suggests the breakdown of feedback regulation from the increased level of intracellular fatty acids. This phenomenon may be derived from increased visceral fat volume and insulin resistance because SREBP-1c and AMPK expression is affected by adipocytokines, insulin signaling and glucose concentration $(4,7)$. However, many NAFLD patients are not obese and do not exhibit increased visceral fat volume or insulin resistance. It has also been demonstrated that the activity of SREBPs, which are produced in the endoplasmic reticulum (ER) as membrane-bound precursors and which form a complex with SREBP-cleavage activating protein (Scap), is post-transcriptionally regulated by intracellular cholesterol levels (7-10). In other words, when cholesterol levels in the ER are too low, Scap escorts SREBPs to the Golgi apparatus where the SREBPs are proteolytically processed to yield active fragments that can enter the nucleus and act as transcriptional factors. When intracellular cholesterol levels are sufficient, the SREBP/Scap complex is held in the ER by insulin-induced genes (Insigs), and the production of active SREBP fragments is inhibited. Moreover, SREBP-1c gene expression is positively regulated by the liver $\mathrm{X}$ receptor $\alpha(\operatorname{LXR} \alpha)$, whose activator ligands are oxysterols (1-14).

Considering the above, hepatic cholesterol metabolism, which has not been extensively investigated in NAFLD patients, needed to be examined in detail. We previously compared the nutritional characteristics between obese and non-obese NAFLD patients and found that dietary over-intake of cholesterol might be a critical factor for the induction and progression of NAFLD (15). Therefore, in the present study, we examined the expression of cholesterol metabolismassociated genes in NAFLD liver and investigated the role of cholesterol metabolism in the onset of NAFLD.

\section{Patients and methods}

Patients and samples for real-time PCR. For real-time PCR, sample tissue was obtained by liver biopsy from 33 patients (male/female 16/17) with histologically diagnosed NAFLD, including 14 with NASH, who were admitted to the Kyushu University Hospital and Kyushu Medical Center between 2005 and 2007. Written informed consent was obtained from all the patients for this investigation.

For the analysis of LXR $\alpha$ and acyl-CoA: cholesterol acyltransferase 1 (ACAT1) expression, patients were divided into obese $(n=23$, male/female 11/12) and non-obese $(n=10$, male/female 5/5) groups according to a body mass index (cut off point: $25 \mathrm{~kg} / \mathrm{m}^{2}$ ). As a control, normal liver tissue was obtained from 10 living donors of liver transplantation whose liver function tests and histological findings were completely normal. We performed real-time RT-PCR and compared gene expression between the NAFLD and normal liver (100\%).

Total RNA was prepared with TRIzol reagent (Invitrogen, Carlsbad, CA, USA), and cDNA was synthesized from $1.0 \mu \mathrm{g}$ RNA with GeneAmp ${ }^{\mathrm{TM}}$ RNA PCR (Applied Biosystems, Branchburg, NJ, USA) using random hexamers. Real-time RT-PCR was performed using LightCycler-FastStart DNA Master SYBR Green 1 (Roche, Basel, Switzerland) according to the manufacturer's instructions. The reaction mixture $(20 \mu \mathrm{l})$ contained LightCycler-FastStart DNA Master SYBR Green 1, $4 \mathrm{mM} \mathrm{MgCl} 2,0.5 \mu \mathrm{M}$ of the upstream and downstream PCR primers, and $2 \mu \mathrm{l}$ of first-strand cDNA as a template. To control variations in the reactions, all PCR data were normalized against $B$-actin expression. The PCR primers for amplification of the ATP binding cassette (ABC) G5 were forward 5'-ATTGTGGTTCTCACCATTCACCAG-3' and reverse 5'-GGTTTGAATGTTCAGGACAAGGGTA-3'; the primers for ACAT1 were forward 5'-GCAGCGAAGAGGC TCAATG-3' and reverse 5'-GCAGCATATACAGGAGCAA TTGG-3'; the primers for apoB100 were forward 5'-TCAAG AGTTACAGCAGATCCATCAA-3' and reverse 5'-TCAGA ATGGAAGTCCTTAAGAGCAA-3'; the primers for farnesyldiphosphate synthase (FDPS) were forward 5'-GCATGTA TCTACCGCCTGCTGA-3' and reverse 5'-TCCAGGGTCT GCCCAATCTC-3'; the primers for HMG-CoA reductase (HMGR) were forward 5'-GCCTGGCTCGAAACATCTG AA-3' and reverse 5'-CTGACCTGGACTGGAAACGGA TA-3'; the primers for HMG-CoA synthase (HMGS) were forward 5'-GTATGCCCTGGTAGTTGCAGGAG-3' and reverse 5'-TGTTGCATATGTGTCCCACGAA-3'; the primers for LDL receptor (LDLR) were forward 5'-CAAC GGCTCAGACGAGCAAG-3' and reverse 5'-AGTCACAGA CGAACTGCCGAGA-3'; the primers for LXR $\alpha$ were forward 5'-GCCGAGTTTGCCTTGCTCA-3' and reverse 5'TCCGGAGGCTCACCAGTTTC-3'; the primers for microsomal triglyceride transfer protein (MTP) were forward 5'AGCACCTCAGGACTGCGAAGA-3' and reverse 5'-CAGA GGTGACAGCATCCACCA-3'; the primers for NiemannPick C1 like 1 (NPC1L1) were forward 5'-TCTGTGGAGT TTGTGTCCCACATTA-3' and reverse 5'-GTTGGTCATGG CCACACCTG-3'; the primers for SREBP-2 were forward 5'ACAACCCATAATATCATTGAGAAACG-3' and reverse 5'-TTGTGCATCTTGGCGTCTGT-3'; the primers for squalene synthase (SS) were forward 5'-CGTGCAGTGCC TGAATGAACTTA-3' and reverse 5'-GGCAGCCAAAGTG GCAATG-3'; the primers for $\beta$-actin were forward 5'-TGGC ACCCAGCACAATGAA-3' and reverse 5'-CTAAGTCAT AGTCCGCCTAGAAGCA -3'.

Statistical analysis. For statistical analysis, continuous variables were compared using the Mann-Whitney U-test, and categorical variables were compared using the Chi-square test or Fisher's test. $\mathrm{P}<0.05$ was considered statistically significant. The results are expressed as means $\pm \mathrm{SD}$.

\section{Results}

Expression pattern of LXRa and ACAT1. Previously, we reported that, in NAFLD patients, the expression of SREBP$1 \mathrm{c}$ and LXR $\alpha$ was highly increased and that up-regulation of LXR $\alpha$ was closely associated with SREBP-1c overexpression (6). LXR $\alpha$ is a sterol sensor, and increased intracellular cholesterol drives the production of oxysterols, which are agonists for LXR $\alpha$ (7). Because cholesterol over-intake is more characteristic of non-obese NAFLD patients (15), mRNA expression of LXR $\alpha$ was re-evaluated by real-time PCR and compared between the obese and non-obese NAFLD patients (see Patients and methods). The characteristics of the patients are shown in Table I. As a result, the LXR $\alpha$ levels 
Table I. Background of the patients.

\begin{tabular}{lccc}
\hline & Obese & Non-obese & \\
\hline Body mass index $\left(\mathrm{kg} / \mathrm{m}^{2}\right)$ & $29.6 \pm 2.8$ & $23.6 \pm 1.7$ & $\mathrm{p}<0.01$ \\
Gender $(\mathrm{M} / \mathrm{F})$ & $21 / 21$ & $5 / 7$ & \\
Age $($ years $)$ & $51.2 \pm 14.0$ & $47.2 \pm 15.1$ & \\
Waist circumference $(\mathrm{cm})$ & $98.6 \pm 8.8$ & $85.1 \pm 5.7$ & $\mathrm{p}<0.01$ \\
Visceral fat area $\left(\mathrm{cm}^{2}\right)$ & $111.3 \pm 32.5$ & $79.2 \pm 39.8$ & \\
Alanine aminotransferase $(\mathrm{IU} / \mathrm{l})$ & $83.8 \pm 55.6$ & $72.4 \pm 48.4$ & \\
$\gamma$-glutamyl transpeptidase $(\mathrm{IU} / \mathrm{l})$ & $67.8 \pm 53.6$ & $60.9 \pm 16.9$ & \\
Total cholesterol $(\mathrm{mg} / \mathrm{dl})$ & $207.9 \pm 36.1$ & $229.9 \pm 29.6$ & \\
Triglycerides $(\mathrm{mg} / \mathrm{dl})$ & $169.9 \pm 116.8$ & $134.4 \pm 47.9$ & \\
HOMA-IR & $4.4 \pm 3.3$ & $2.4 \pm 1.2$ & \\
Adiponectin $(\mu \mathrm{g} / \mathrm{dl})$ & $6.67 \pm 2.67$ & $6.71 \pm 2.42$ & \\
TNF- $\alpha(\mathrm{pg} / \mathrm{ml})$ & $1.28 \pm 0.52$ & $1.38 \pm 0.92$ & \\
\hline
\end{tabular}

were significantly higher in the non-obese group although the levels in the obese group were also elevated by more than 3 -fold compared with those in the control group (Fig. 1A). Moreover, we also found that the expression of ACAT1, an enzyme associated with esterification of cholesterol, was greater in NAFLD liver than in normal liver and that the nonobese patients showed greater expression of ACAT1 than the obese patients (Fig. 1B).

Expression of cholesterol metabolism-associated genes in NAFLD. To analyze the cholesterol metabolism in NAFLD liver, the expression levels of cholesterol metabolism-
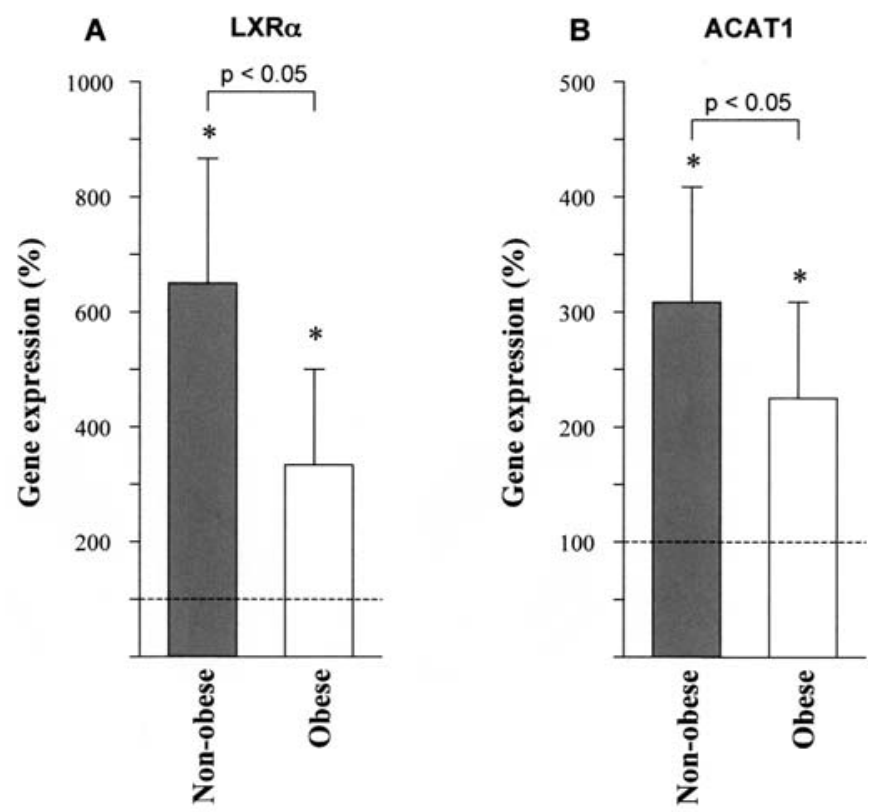

Figure 1. Real-time RT-PCR analysis of LXR $\alpha$ (A) and ACAT1 (B) gene expression in NAFLD liver. LXR $\alpha$ and ACAT1 expression was significantly higher in the non-obese group (BMI $<25 \mathrm{~kg} / \mathrm{m}^{2}$ ) than that in the obese group $\left(\right.$ BMI $\left.>25 \mathrm{~kg} / \mathrm{m}^{2}\right)$. *Statistically significant differences $(\mathrm{p}<0.05)$ compared with normal liver $(100 \%)$. LXR $\alpha$, liver X receptor $\alpha$; ACAT1, acyl-CoA: cholesterol acyltransferase 1 . associated genes were evaluated by real-time PCR and compared between the NAFLD and normal control groups (Fig. 2). In NAFLD, the expression levels of LDLR and SREBP-2 were greatly reduced by 90 and $50 \%$, respectively. In contrast, the expression levels of apoB100 and MTP were enhanced by nearly 4- and 2-fold, respectively. Furthermore, expression of the ABCG5 was increased by nearly 3-fold, whereas the expression of NPC1L1 was unchanged. Gene expression levels of cholesterol synthesis-associated enzymes were also estimated (Fig. 3). The expression of HMGR, which is the first committed enzyme in the mevalonate pathway, was enhanced by nearly 2.5-fold despite low expression of LDLR and SREBP-2. The expression patterns of the other enzymes tested, HMGS, FDPS, and SS, were broadly similar to that of HMGR. Fig. 4 shows a schema for the regulation of cholesterol in hepatocytes.

\section{Discussion}

It is well known that the onset and progression of NAFLD is closely associated with increased visceral fat volume, insulin resistance and hepatocytic accumulation of fatty acids and triglycerides, resulting in oxidative stress (16). In obese NAFLD patients, such findings are relatively common. Fatty acid synthesis in hepatocytes is positively regulated by the transcriptional factor SREBP-1c (7). When intracellular fatty acid levels go up, AMPK, a negative regulator of lipogenesis, down-regulates SREBP-1c. Oberkofler et al reported that, in adipose tissue, SREBP-1c mRNA expression in obese humans was lower than that in non-obese subjects and that the expression was up-regulated after weight loss (17). On the other hand, in our previous study, SREBP-1c expression levels were 5-fold higher in the liver of NAFLD patients compared with healthy controls, and the up-regulated SREBP-1c

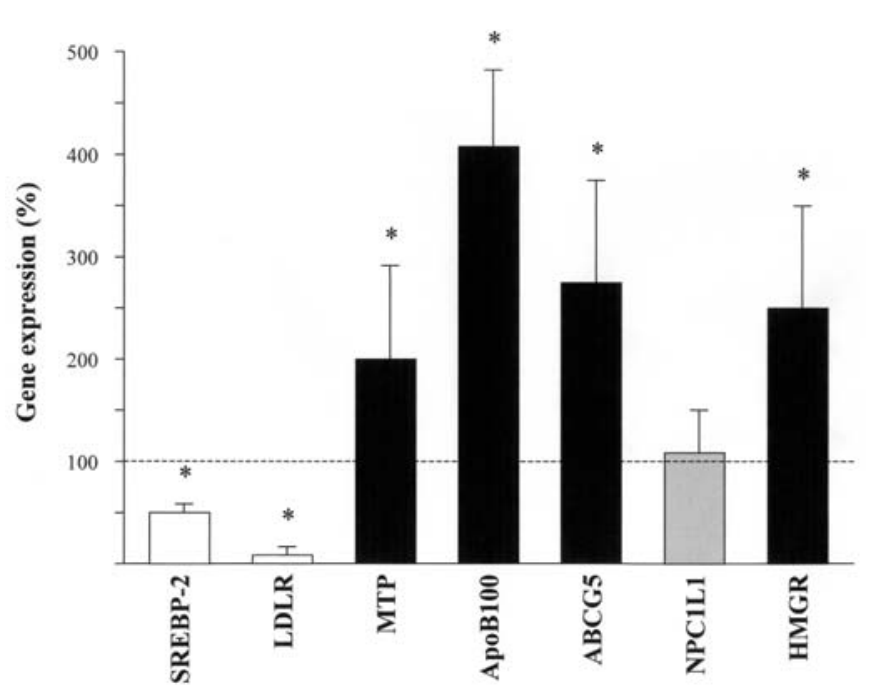

Figure 2. Real-time RT-PCR analysis of SREBP-2, LDLR, MTP, apoB100, ABCG5, NPC1L1 and HMGR gene expression in NAFLD liver. *Statistically significant differences $(\mathrm{p}<0.05)$ compared with normal liver (100\%). SREBP-2, sterol regulatory element-binding protein-2; LDLR, LDL receptor; MTP, microsomal triglyceride transfer protein; ABCG5, ATP binding cassette G5; NPC1L1, Niemann-Pick C1 like 1; HMGR, HMG-CoA reductase. 
A

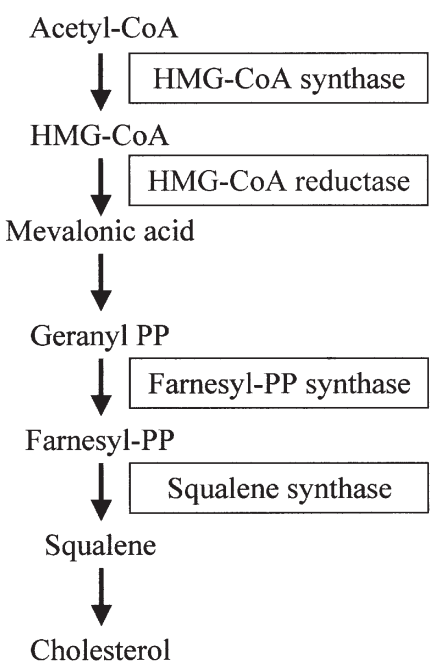

B

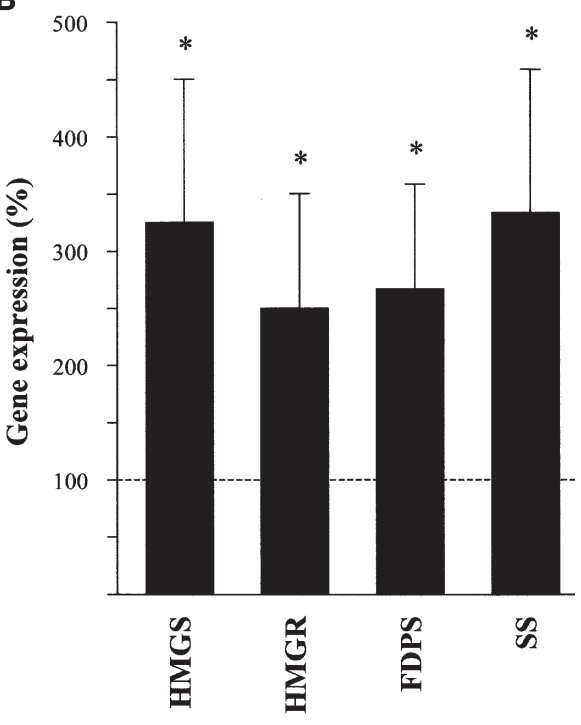

Figure 3. (A) Cholesterol synthesis pathway in hepatocytes and its related enzymes. (B) Real-time RT-PCR analysis of HMG-CoA reductase (HMGR), HMGCoA synthase (HMGS), farnesyl-diphosphate synthase (FDPS), and squalene synthase (SS) gene expression in NAFLD liver. ${ }^{*}$ Statistically significant differences $(\mathrm{p}<0.05)$ compared with normal liver $(100 \%)$.

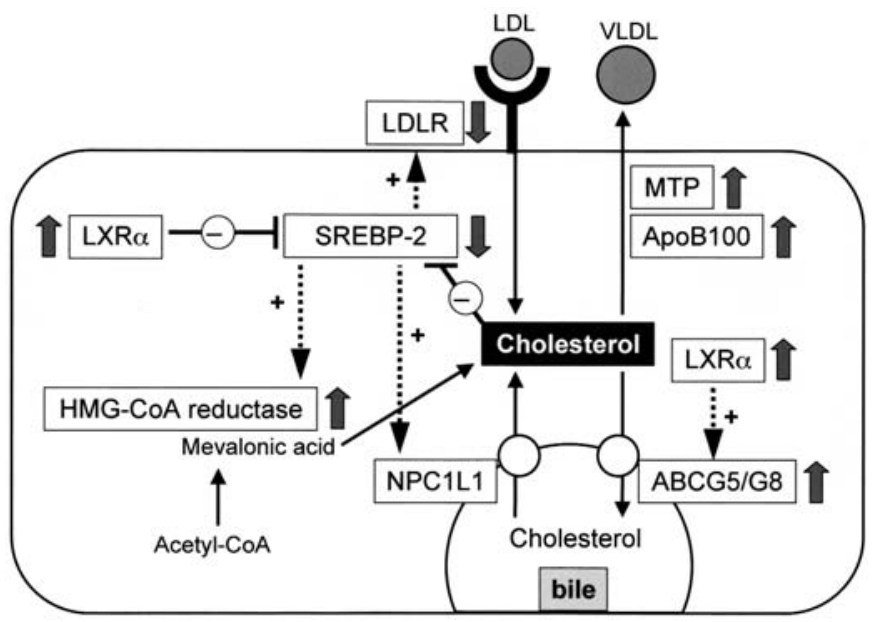

Figure 4. Schema of cholesterol metabolism in hepatocytes. Wide gray arrows indicate changes in gene expression in NAFLD liver. ABCG5/G8, ATP binding cassette G5/G8; LDLR, LDL receptor; LXR $\alpha$, liver X receptor $\alpha$; MTP, microsomal triglyceride transfer protein; NPC1L1, Niemann-Pick C1 like 1; SREBP-2, sterol regulatory element-binding protein-2.

contributes to increased de novo synthesis of fatty acids, even in hepatocytes with fatty acid accumulation (2-6). Upregulated insulin receptor substrate-1 (IRS-1) is associated with up-regulation of SREBP-1c, and suppression of SREBP-1c by AMPK fails in NAFLD (2-6). However, many NAFLD patients are not obese and many of them do not exhibit insulin resistance or increased visceral fat volume (Table I). These data compel us to question what triggers and maintains the disease in non-obese patients. To explore this problem, we examined the nutritional assessment of NAFLD patients and compared findings between obese and non-obese patients (15). Unsurprisingly, total energy and carbohydrate intake was significantly higher in obese patients. Interestingly, a characteristic finding was that dietary cholesterol intake was significantly higher in non-obese patients than in obese patients whose cholesterol intake was also increased compared with normal controls (15). This is an important clue since LXR $\alpha$, a sterol sensor that regulates intracellular cholesterol levels, can induce SREBP-1c expression and activation (7).

It has been reported that SREBP-1c is positively regulated through the transcription factor LXR $\alpha$ which forms a heterodimer with retinoid $X$ receptor $\alpha$ (18). Since stimulation of the peroxisome proliferator activated receptor $\alpha(\operatorname{PPAR} \alpha)$ increases LXR $\alpha$ expression $(19,20)$, the accumulated fatty acids in the NAFLD liver, which are endogenous PPAR $\alpha$ agonists, may increase LXR $\alpha$ expression. In addition, insulin increases LXR $\alpha$ expression (21); therefore, the lipogenic effect of insulin in the NAFLD liver may be partially mediated via the LXR $\alpha$ pathway. LXR $\alpha$ expression is controlled by autoregulation because the $\mathrm{LXR} \alpha$ gene promoter contains functional LXR response elements (22). Ligands of $\operatorname{LXR} \alpha$ include the cholesterol derivatives oxysterols. We previously reported that $\mathrm{LXR} \alpha$ expression is highly up-regulated, in addition to SREBP-1c, and shows a strong positive correlation with SREBP-1c in NAFLD patients (6). Moreover, in the non-obese group, whose dietary cholesterol intake was greater than in the obese group, the LXR $\alpha$ levels were significantly higher than in the obese group (Fig. 1A). These results indicate that cholesterol excess, which ultimately results in elevated oxysterols, stimulates SREBP-1c synthesis through an LXR $\alpha$-dependent mechanism, particularly in nonobese NAFLD patients. SREBP-1c may be primarily upregulated by $\mathrm{LXR} \alpha$ in NAFLD although, as shown in our previous reports (2-6), insulin signaling and intracellular glucose levels also affect SREBP-1c expression. We assumed that the up-regulation of SREBP-1c is mainly attributable to the activation of $\mathrm{LXR} \alpha$ for which the natural ligands are oxysterols. 
Nevertheless, it may be that cholesterol itself and certain oxysterols inhibit proteolytic processing of the inactive SREBP-1c precursor to its mature active form, which works in the nucleus (8). However, the post-transcriptional regulation at sufficient intracellular cholesterol levels may be considered as a transient short-time regulation under physiological situations; therefore, it is reasonable to assume that the posttranscriptional mechanism cannot function appropriately in chronic diseases such as NAFLD. Accordingly, cholesterol excess seems to be positively associated with the initiation and progression of NAFLD. Indeed, in animal models, it has been reported that a high cholesterol diet causes NASH without obesity (23-25). Therefore, it is plausible that a person with a high cholesterol intake could develop non-obese NAFLD. Up-regulation of LXR $\alpha$ is needed to regulate intracellular cholesterol levels; however, it also up-regulates lipogenesis via SREBP-1c resulting in steatosis, which is apparently deleterious for hepatocytes. Generally, most intracellular cholesterol is esterified with fatty acids by ACAT1. In our study, ACAT1 expression was greater in NAFLD, particularly in non-obese patients than in the normal controls (Fig. 1B). Therefore, it is likely that an abundance of cholesterol promotes fatty acid production for its esterification.

Next, we evaluated cholesterol metabolism in the NAFLD liver. Cholesterol metabolism can occur via three mechanisms; first, as we have shown, the cholesterol-LXR $\alpha$-SREBP-1c signaling pathway leading to fatty acid synthesis; second, de novo cholesterol synthesis, its uptake from serum, and output into serum or bile; and third, bile acid synthesis and its secretion. Our question was whether physiological cholesterol metabolism is disturbed or not in NAFLD liver. Serum LDL-cholesterol is taken into hepatocytes via the LDLR. For secretion, cholesterol is packed into lipoproteins together with triglycerides and apoB 100 by MTP, and secreted into serum as VLDL. Cholesterol is also secreted into bile via ABCG5/ABCG8. SREBP-2 is a key factor for cholesterol synthesis, which simultaneously stimulates gene expression of LDLR and HMGR. The accumulation of cholesterol in hepatocytes and LXR $\alpha$ combine to downregulate SREBP-2 activity, and thus decrease cholesterol synthesis and uptake (7).

In our study, SREBP-2 and LDLR expression was downregulated in NAFLD liver (Fig. 2), which could be explained by cholesterol overload and LXR $\alpha$ activation. Surprisingly, the expression of HMGR showed a contradictory result and was enhanced by nearly 2.5-fold (Fig. 3), despite the low expression levels of LDLR and SREBP-2. Moreover, the expression of other enzymes associated with cholesterol synthesis such as HMGS, FDPS and SS, was up-regulated in NAFLD liver (Fig. 3). These data indicate that the regulation of cholesterol synthesis and uptake is impaired in NAFLD liver. At present, we cannot explain the exact reason for this discrepancy. More studies are needed for the precise understanding of the system that regulates cholesterol. The increased expression of apoB100 and MTP in NAFLD liver (Fig. 2) may be involved in the response to overload of cholesterol and triglycerides with VLDL secretion. ABCG5 gene expression is increased by a high cholesterol diet and is mediated by the LXR $\alpha$ pathway (26-29). Cholesterol loading of hepatocytes results in the increased formation of oxysterols, which stimulate LXR $\alpha$ and enhance cholesterol efflux into bile through ABCG5/ABCG8 transporters. Therefore, upregulation of ABCG5 expression in our study is an expected result, while NPC1L1 expression was unchanged between NAFLD and normal liver (Fig. 2).

NPC1L1 is a sterol transporter that is present in the proximal jejunum and on the canalicular aspect of hepatocytes (26). It is essential for the absorption/ reabsorption of cholesterol and of other plant sterols from the intestine and liver. In animal models, it has been reported that knockout of NPC1L1 or treatment with ezetimibe, an inhibitor of NPC1L1-dependent cholesterol transport, provides resistance against hepatic steatosis $(30,31)$. With regard to treatment options, lowering of intrahepatic cholesterol levels may be beneficial for treating NAFLD. From this point of view, ezetimibe and statins, HMGR inhibitors, can be used as a new therapeutic option for NAFLD. It is expected that the inhibition of NPC1L1-dependent absorption of dietary and biliary cholesterol will decrease intracellular cholesterol levels and down-regulate/inactivate $\mathrm{LXR} \alpha$, resulting in an improvement of steatohepatitis. We are now running a pilot study of ezetimibe to treat NAFLD patients. Although only a preliminary result, ezetimibe significantly lowered the serum levels of alanine aminotransferase and $\gamma$-glutamyl transpeptidase after administration for 4 months (data not shown).

In this study, we obtained the following findings in NAFLD patients. i) Cholesterol over-intake is a potent inducer of NAFLD, which is primarily achieved via the LXR $\alpha$ SREBP-1c pathway. ii) LXR $\alpha$ was overexpressed in the liver of NAFLD patients, and the expression was positively correlated with SREBP-1c expression at least at mRNA levels. iii) Physiological cholesterol metabolism in the liver may be, at least partially, disturbed in NAFLD liver. iv) Some modulators of cholesterol metabolism, such as ezetimibe and statins, may have therapeutic potential for NAFLD.

\section{References}

1. Angulo P: Nonalcoholic fatty liver disease. N Engl J Med 346: 1221-1231, 2002.

2. Nakamuta M, Kohjima M, Morizono S, Kotoh K, Yoshimoto T, Miyagi I and Enjoji M: Evaluation of fatty acid metabolismrelated gene expression in nonalcoholic fatty liver disease. Int J Mol Med 16: 631-635, 2005.

3. Kohjima M, Enjoji M, Higuchi N, Katoh M, Kotoh K, Yoshimoto T, Fujino T, Yada M, Yada R, Harada N, Takayanagi $\mathrm{R}$ and Nakamuta M: Re-evaluation of fatty acid metabolism-related gene expression in nonalcoholic fatty liver disease. Int J Mol Med 20: 351-358, 2007.

4. Kohjima M, Higuchi N, Katoh M, Kotoh K, Yoshimoto T, Fujino T, Yada M, Yada R, Harada N, Enjoji M, Takayanagi R and Nakamuta M: SREBP-1c, regulated by the insulin and AMPK signaling pathways, plays a role in nonalcoholic fatty liver disease. Int J Mol Med 21: 507-511, 2008.

5. Kato M, Higuchi N and Enjoji M: Reduced expression of ATGL and CGI-58 in the liver may attribute to develop NAFLD in patients with insulin resistant background. Scand J Gastroenterol 43: 1018-1019, 2008.

6. Higuchi N, Kato M, Syundo N, Tajiri H, Tanaka M, Yamashita N, Kohjima M, Kotoh K, Nakamuta M, Takayanagi R and Enjoji M: Liver X receptor in cooperation with SREBP-1c is a major lipid synthesis regulator in nonalcoholic fatty liver disease. Hepatol Res 38: 1122-1129, 2008

7. Wójcicka G, Jamroz-Wisniewska A, Horoszewicz K and Beltowski J: Liver X receptors (LXRs). Part I: Structure, function, regulation of activity, and role in lipid metabolism. Postepy Hig Med Dosw 61: 736-759, 2007. 
8. Goldstein JL, DeBose-Boyd RA and Brown MS: Protein sensors for membrane sterols. Cell 124: 35-46, 2006.

9. Gill S, Chow R and Brown AJ: Sterol regulators of cholesterol homeostasis and beyond: the oxysterol hypothesis revisited and revised. Progress Lipid Res 47: 391-404, 2008.

10. Radhakrishnan A, Ikeda Y, Kwon HJ, Brown MS and Goldstein JL: Sterol-regulated transport of SREBPs from endoplasmic reticulum to Golgi: oxysterols block transport by binding to Insig. Proc Natl Acad Sci USA 104: 6511-6518, 2007.

11. Engelking LJ, Liang G, Hammer RE, Takaishi K, Kuriyama H, Evers BM, Li W-P, Horton JD, Goldstein JL and Brown MS: Schoenheimer effect explained - feedback regulation of cholesterol synthesis in mice mediated by Insig proteins. J Clin Invest 115: 2489-2498, 2005.

12. Zelcer $\mathrm{N}$ and Tontonoz P: Liver $\mathrm{X}$ receptors as integrators of metabolic and inflammatory signaling. J Clin Invest 116 : 607-614, 2006.

13. Cha J-Y and Repa JJ: The liver X receptor (LXR) and hepatic lipogenesis. J Biol Chem 282: 743-751, 2007.

14. Repa JJ and Mangelsdorf DJ: The liver X receptor gene team: potential new players in atherosclerosis. Nat Med 8: 1243-1248, 2002.

15. Yasutake K, Nakamuta M, Shima Y, Ohyama A, Masuda K, Haruta N, Fujino T, Aoyagi Y, Fukuizumi K, Yoshimoto T, Takemoto R, Miyahara T, Harada N, Hayata F, Sakai Y, Nakashima $M$ and Enjoji M: Nutritional investigation of nonobese patients with non-alcoholic fatty liver disease: the significance of dietary cholesterol. Scand J Gastroenterol 4: 107, 2008.

16. Saito T, Misawa K and Kawata S: Fatty liver and non-alcoholic steatohepatitis. Intern Med 46: 101-103, 2007.

17. Oberkofler H, Fukushima N, Esterbauer H, Krempler F and Patsch W: Sterol regulatory element binding proteins: relationship of adipose tissue gene expression with obesity in humans. Bioochim Biophys Acta 1575: 75-81, 2002.

18. Chen G, Liang G, Ou J, Goldstein JL and Brown MS: Central role for liver X receptor in insulin-mediated activation of Srebp$1 \mathrm{c}$ transcription and stimulation of fatty acid synthesis in liver. Proc Natl Acad Sci USA 101: 11245-11250, 2004.

19. Laffitte BA, Joseph SB, Walczak R, Pei L, Wilpitz DC, Collins JL and Tontonoz P: Autoregulation of the human liver X receptor $\alpha$ promoter. Mol Cell Biol 21: 7558-7568, 2001.

20. Tobin KA, Steineger HH, Alberti S, Spydevold O, Auwerx J, Gustafsson JA and Nebb HI: Cross-talk between fatty acid and cholesterol metabolism mediated by liver X receptor $\alpha$. Mol Endocrinol 14: 741-752, 2000.

21. Tobin KA, Ulven SM, Schuster GU, Steineger HH, Andresen SM, Gustafsson JA and Nebb HI: Liver X receptor $\alpha$ as insulinmediating factors in fatty acid and cholesterol biosynthesis. J Biol Chem 277: 10691-10697, 2002.
22. Li Y, Bolten C, Bhat BG, Woodring-Dietz J, Li S, Prayaga SK, $\mathrm{Xia} C$ and Lala DS: Induction of human liver $\mathrm{X}$ receptor $\alpha$ gene expression via an autoregulatory loop mechanism. Mol Endocrinol 16: 506-514, 2002.

23. Jeong WI, Jeong DH, Do SH, Kim YK, Park HY, Kwon OD, Kim TH and Jeong KS: Mild hepatic fibrosis in cholesterol and sodium cholate diet-fed rats. J Vet Med Sci 67: 235-242, 2005.

24. Kainuma M, Fujimoto M, Sekiya N, Tsuneyama K, Cheng C, Takano Y, Terasawa K and Shimada Y: Cholesterol-fed rabbit is a unique model of nonalcoholic, non-obese, non-insulinresistant fatty liver disease with characteristic fibrosis. J Gastroenterol 41: 971-980, 2006.

25. Matsuzawa N, Takamura T, Kurita S, Misu H, Ota T, Ando H, Yokoyama M, Honda M, Zen Y, Nakanuma Y, Miyamoto K and Kaneko S: Lipid-induced oxidative stress causes steatohepatitis in mice fed an atherogenic diet. Hepatology 46: 1392-1403, 2007.

26. Kidambi S and Patel SB: Cholesterol and non-cholesterol sterol transporters: ABCG5, ABCG8 and NPC1L1: a review. Xenobiotica 38: 1119-1139, 2008.

27. Yu L, York J, von Bergmann K, Lutjohann D, Cohen JC and Hobbs HH: Stimulation of cholesterol excretion by the liver $\mathrm{X}$ receptor agonist requires ATP-binding cassette transporters G5 and G8. J Biol Chem 278: 15565-15570, 2003.

28. Berge KE, Tian H, Graf GA, Yu L, Grishin NV, Schultz J, Kwiterovich P, Shan B, Barnes R and Hobbs HH: Accumulation of dietary cholesterol in sitosterolemia caused by mutations in adjacent ABC transporters. Science 290: 1771-1775, 2000.

29. Rapa JJ, Berge KE, Pomajzl C, Richardson JA, Hobbs HH and Mangeladorf DJ: Regulation of ATP-binding cassette sterol transporters ABCG5 and ABCG8 by the liver X-receptors alpha and beta. J Biol Chem 277: 18793-18800, 2002.

30. Davies JP, Scott C, Oishi K, Liapis A and Ioannou YA: Inactivation of NPC1L1 causes multiple lipid transport defects and protects against diet-induced hypercholesterolemia. J Biol Chem 280: 12710-12720, 2005.

31. Deushi M, Nomura M, Kawakami A, Haraguchi M, Ito M, Okazaki M, Ishii $\mathrm{H}$ and Yoshida M: Ezetimibe improves liver steatosis and insulin resistance in an obese rat model of the metabolic syndrome. FEBS Lett 581: 5664-5670, 2007. 